\title{
Targeting vulnerable atherosclerotic plaque via PET-tracers aiming at cell-surface overexpression of somatostatin receptors
}

\author{
GEORGIOS Z. PAPADAKIS ${ }^{1,2}$, GEORGE KOCHIADAKIS ${ }^{3}$, GEORGE LAZOPOULOS ${ }^{4}$, \\ KOSTAS MARIAS $^{2}$, NIKOLAOS KLAPSINOS ${ }^{2}$, FADY HANNAH-SHMOUNI ${ }^{5}$, \\ GEORGIA G. IGOUMENAKI ${ }^{2}$, TAXIARCHIS KONSTANTINOS NIKOLOUZAKIS ${ }^{6}$, \\ STELIOS KTENIADAKIS ${ }^{7}$, DEMETRIOS A. SPANDIDOS $^{8}$ and APOSTOLOS H. KARANTANAS ${ }^{1,2}$ \\ ${ }^{1}$ Department of Radiology, Medical School, University of Crete, 71003 Heraklion; \\ ${ }^{2}$ Foundation for Research and Technology Hellas (FORTH), Computational Biomedicine Laboratory (CBML), \\ 70013 Heraklion; ${ }^{3}$ Cardiology Department, University of Crete, 71110 Heraklion; ${ }^{4}$ Department of \\ Cardiothoracic Surgery, University General Hospital of Heraklion, University of Crete, Medical School, \\ 71003 Heraklion, Greece; ${ }^{5}$ Internal Medicine-Endocrinology, Hypertension and Metabolic Genetics, \\ Section on Endocrinology and Genetics, Eunice Kennedy Shriver National Institute of Child Health and \\ Human Development, National Institutes of Health, Bethesda, MD 20892, USA; ${ }^{6}$ Laboratory of \\ Anatomy-Histology-Embryology, Medical School, University of Crete, 71003 Heraklion; \\ ${ }^{7}$ Emergency Department, Venizeleion General Hospital, 71409 Heraklion; ${ }^{8}$ Laboratory of \\ Clinical Virology, Medical School, University of Crete, 70013 Heraklion, Greece
}

Received May 25, 2020; Accepted June 15, 2020

DOI: 10.3892/br.2020.1316

\begin{abstract}
Cardiovascular disease (CD) is the leading cause of death in the developed world, with major atherothrombotic events, being mainly attributed to the rupture of unstable, vulnerable atherosclerotic lesions, leading to blood flow obstruction. Since unstable atherosclerotic plaques frequently do not cause hemodynamically significant blood flow restriction, conventional stress imaging tests cannot depict the vulnerable, high-risk for rupture atherosclerotic lesions. Therefore, molecular imaging techniques targeting specific pathophysiologic features related to atherosclerotic plaque rupture mechanism, hold promise for precise and individualized treatment strategies of $\mathrm{CD}$. In the current report, we describe in a patient diagnosed with pancreatic neuroendocrine
\end{abstract}

Correspondence to: $\mathrm{Dr}$ Georgios Z. Papadakis, Foundation for Research and Technology Hellas (FORTH), Computational Biomedicine Laboratory (CBML), 100 N. Plastira, Vassilika Vouton, 70013 Heraklion, Greece

E-mail: gzpapadakis@gmail.com

Abbreviations: $\mathrm{CD}$, cardiovascular disease; NET, neuroendocrine tumor; PET, positron emitting tomography; CT computed tomography; LDL, low-density lipoproteins; SSTRs, somatostatin receptors; VWI, vessel wall inflammation

Key words: atherosclerosis, vulnerable plaque; vessel wall inflammation, ${ }^{68} \mathrm{Ga}$-DOTATATE PET/CT, somatostatin receptors, pancreatic neuroendocrine tumor tumor, the selective uptake of ${ }^{68} \mathrm{Ga}$-DOATATE by an atherosclerotic lesion in the thoracic aorta. This data indicates that ${ }^{68} \mathrm{Ga}$-DOTATATE, which is a positron emitting tomography tracer, targeting the recruitment of macrophages taking place in the vulnerable plaque, could potentially serve as an imaging probe for the detection of high-risk, prone to rupture plaques.

\section{Introduction}

Despite progress in prevention, diagnosis and therapy, cardiovascular disease (CD) stands for the leading cause of morbidity and mortality in the developed world (1). The majority of CD-related deaths are due to acute thrombotic events, following the rupture of atherosclerotic lesions, which are characterized by key pathophysiologic features. The capability of positron emitting tomography (PET) imaging to visualize and quantify these features at the cellular and sub-cellular level provides the ground for the employment of PET-radiopharmaceuticals, which target rupture-related biochemical processes, in order to address the challenge of detecting high-risk vulnerable atherosclerotic lesions.

The pathophysiology of atherosclerosis is quite complex, and is mainly characterized by an inflammatory cascade triggered by the entrance of low-density lipoproteins (LDL) at sites of endothelial injury, and the subsequent recruitment of macrophages which take up the oxidized LDL remnants (2). An extensive description of the atherosclerotic plaque pathophysiology is beyond the scope of the current report. Our main interest is focused on the key role that infiltration by macrophages plays in inflammatory processes encountered in unstable atherosclerotic plaques. 
Since the expression of somatostatin receptors (SSTRs) subtype- 2 , has been detected on macrophages $(3,4)$, these cells can be effectively targeted with somatostatin analogues radio-labelled with isotopes suitable for PET-imaging. Such PET-tracers, which enable whole-body characterization of cell surface SSTRs-expression, have become the imaging standard of reference for the detections of neuroendocrine tumors (NETs) and other SSTRs-positive lesions (5-12). Furthermore, we have previously reported the increased uptake of ${ }^{68} \mathrm{Ga}$-DOTATATE, which is one of the commercially available PET-tracers targeting cell surface SSTRs-subtype-2-over-expression, at sites of reactive inflammatory alterations $(13,14)$.

\section{Case report}

A 82-year-old man presented with a constant epigastric pain. The computed tomography (CT) scan of the abdomen, showed a large $(3 \mathrm{~cm})$ pancreatic head mass. Subsequently, endoscopic ultrasound (EUS) and biopsy of the tumor revealed a low-grade NET. Therefore, a whole body ${ }^{68} \mathrm{Ga}$-DOTATATE PET/CT scan was performed for staging purposes. The PET/CT study showed (Fig. 1A) intense radiotracer uptake $\left(\mathrm{SUV}_{\max }: 85\right)$ by the pancreatic head tumor and excluded the presence of metastatic disease.

Furthermore, there was extensive atherosclerosis seen throughout medium- and large-sized arteries such as in the abdominal aorta, most of which were ${ }^{68} \mathrm{Ga}$-DOTATATE-negative (Fig. 1B; red arrows). However, in the thoracic aorta a radiotracer-positive plaque $\left(\mathrm{SUV}_{\max }: 5.5\right)$ was encountered (Fig. 1C; yellow arrow), implying infiltration by macrophages, which are known to be characterized by cell-surface over-expression of SSTRs-subtype-2, leading to increased ${ }^{68} \mathrm{Ga}$-DOTATATE activity. At the same level of the thoracic aorta, another ${ }^{68} \mathrm{Ga}$-DOTATATE-negative plaque was detected (Fig. 1C; white arrow), suggesting that not all atherosclerotic lesions take up the administered PET-tracer. Despite its small size, the ${ }^{68} \mathrm{Ga}$-DOTATATE avidity of the plaque seen on the thoracic aorta, suggests an active inflammatory cascade taking place in that specific lesion, raising suspicion for a high-risk prone to rupture lesion.

\section{Discussion}

Early and accurate detection of high-risk, prone to rupture atherosclerotic plaques, is the holy grail of CD research, receiving great interest and extensive research efforts. Molecular imaging of key rupture-related pathophysiological features of the plaques, by means of PET-tracers, holds promise to address this diagnostic challenge. Imaging the recruitment of macrophages at sites of vessel wall inflammation (VWI), via PET-tracers aiming at the cell-surface over-expression of STTRs subtype-2, also seen on macrophages, is a promising molecular imaging strategy for the detection of the unstable plaques.

In a series of 16 patients with NETS, LiX. et al.reported association between coronary artery uptake of ${ }^{68} \mathrm{Ga}$-DOTATATE with known risk factors of CD (15). Furthermore, Pedersen SF et al, in a cohort of 10 patients who underwent simultaneous PET/MRI scans using ${ }^{64} \mathrm{Cu}$-DOTATATE, prior to carotid endarterectomy, found increased tracer uptake in symptomatic plaques, while an independent correlation with CD 163 gene
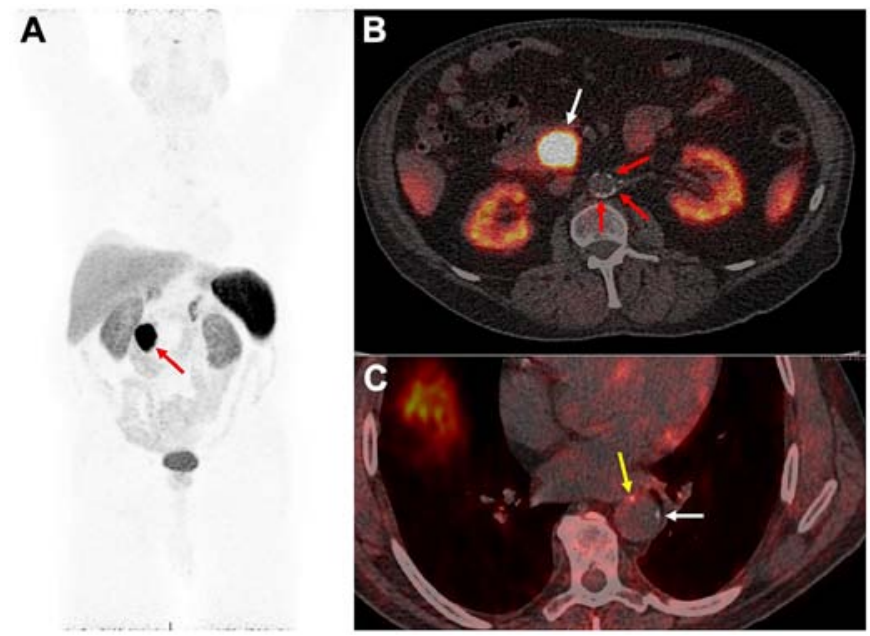

Figure 1. (A) MIP ${ }^{68} \mathrm{Ga}$-DOTATATE PET image of the of the head and torso showing (red arrow) a large tumor in the head of the pancreas with intensely elevated uptake $\left(\mathrm{SUV}_{\max }: 85\right)$. (B) Axial fused ${ }^{68} \mathrm{Ga}$-DOTATATE PET/CT image of the abdomen demonstrating the ${ }^{68} \mathrm{Ga}$-DOTATATE-avid pancreatic head mass (white arrow) and extensive atherosclerotic lesions in the aorta (red arrows) which are ${ }^{68} \mathrm{Ga}$-DOTATATE-negative. (C) Axial fused ${ }^{68} \mathrm{Ga}$-DOTATATE PET/CT image of the thorax showing two aortic atherosclerotic lesions, one of which was ${ }^{68} \mathrm{Ga}$-DOTATATE-negative (white arrow), while the other one (yellow arrow) exhibiting elevated radiotracer uptake $\left(\mathrm{SUV}_{\max }: 5.5\right)$ and thus implying infiltration by macrophages. MIP, maximum intensity projection; PET, positron emitting tomography; CT, computed tomography.

expression (surrogate marker of activated macrophages) was revealed (16). In a series of 42 patients with atherosclerosis, Tarkin et al reported that ${ }^{68} \mathrm{Ga}$-DOTATATE correctly detected culprit arteries in patients with acute coronary syndrome, predicted high-risk coronary CT features and was positively associated with Framingham risk score, implying the employment of the radiotracer as a novel imaging probe for VWI (17). Moreover, ${ }^{68} \mathrm{Ga}$-DOTATATE is superior to ${ }^{18} \mathrm{~F}$-FDG which is the most widely used PET-tracer, targeting metabolic activity, since the lack of physiologic uptake by the myocardium, enables assessment of the coronary arteries.

In accordance to the existing literature, the current report adds to the data that not all atherosclerotic plaques exhibit elevated ${ }^{68} \mathrm{Ga}$-DOTATATE activity, suggesting that only lesions harboring active inflammatory processes and therefore are infiltrated by macrophages, take up the tracer. A major limitation of our report is the lack of histologic analysis of the ${ }^{68} \mathrm{Ga}$-DOTATATE-avid plaque in the thoracic aorta, and the confirmation of macrophages accumulation. However, our work enhances the need for further research efforts being addressed towards employment of this specific molecular imaging strategy for the detection of the vulnerable atherosclerotic plaque.

\section{Acknowledgements}

Not applicable.

\section{Funding}

Part of this study was financially supported by the Stavros Niarchos Foundation within the framework of the project 
ARCHERS (Advancing Young Researchers' Human Capital in Cutting Edge Technologies in the Preservation of Cultural Heritage and the Tackling of Societal Challenges).

\section{Availability of data and materials}

All the information relevant to the present study is available from the corresponding author on reasonable request.

\section{Authors' contributions}

GZP, AHK, and GK conceived and designed the study. GZP, GL, KM, NK, FHS, GGI, TKN and SK researched the literature, performed interpretation of data and drafted the manuscript. DAS, and AHK critically revised the article for important intellectual content, and assisted in the literature search for this case report. All authors agree to be accountable for all aspects of the work in ensuring that questions related to the accuracy or integrity of any part of the work are appropriately investigated, and finally approved the version of the manuscript to be published.

\section{Ethics approval and consent to participate}

The images were provided by esteemed physicians at the $\mathrm{NIH}$, with whom the first and corresponding author of the article collaborates. All study participants at the NIH clinical protocols provided all the extensive consent forms and strict ethical approval documents that the NIH standards require.

\section{Patient consent for publication}

Not applicable.

\section{Competing interests}

DAS is the Editor-in-Chief for the journal, but had no personal involvement in the reviewing process, or any influence in terms of adjudicating on the final decision, for this article. All the other authors declare that they have no competing interests.

\section{References}

1. Bucerius J, Dijkgraaf I, Mottaghy FM and Schurgers LJ: Target identification for the diagnosis and intervention of vulnerable atherosclerotic plaques beyond 18F-fluorodeoxyglucose positron emission tomography imaging: Promising tracers on the horizon. Eur J Nucl Med Mol Imaging 46: 251-265, 2019.

2. Krishnan S, Otaki Y, Doris M, Slipczuk L, Arnson Y, Rubeaux M, Dey D, Slomka P, Berman DS and Tamarappoo B: Molecular imaging of vulnerable coronary plaque: A pathophysiologic perspective. J Nucl Med 58: 359-364, 2017.

3. Dalm VA, van Hagen PM, van Koetsveld PM, Achilefu S, Houtsmuller AB, Pols DH, van der Lely AJ, Lamberts SW and Hofland LJ: Expression of somatostatin, cortistatin, and somatostatin receptors in human monocytes, macrophages, and dendritic Cells. Am J Physiol Endocrinol Metab 285: E344-E353, 2003.
4. Armani C, Catalani E, Balbarini A, Bagnoli P and Cervia D: Expression, pharmacology, and functional role of somatostatin receptor subtypes 1 and 2 in human macrophages. J Leukoc Biol 81: 845-855, 2007.

5. Hofman MS, Lau WF and Hicks RJ: Somatostatin receptor imaging with ${ }^{68} \mathrm{Ga}$ DOTATATE PET/CT: Clinical utility, normal patterns, pearls, and pitfalls in interpretation. Radiographics 35: 500-516, 2015

6. Tirosh A, Papadakis GZ, Millo C, Hammoud D, Sadowski SM, Herscovitch P, Pacak K, Marx SJ, Yang L, Nockel P, et al: Prognostic utility of total ${ }^{68} \mathrm{Ga}$-DOTATATE-avid tumor volume in patients with neuroendocrine tumors. Gastroenterology 154: 998-1008.e1, 2018.

7. Tirosh A, Papadakis GZ, Millo C, Sadowski SM, Herscovitch P, Pacak K, Marx SJ, Yang L, Nockel P, Shell J, et al: Association between neuroendocrine tumors biomarkers and primary tumor site and disease type based on total ${ }^{68} \mathrm{Ga}$-DOTATATE-Avid tumor volume measurements. Eur J Endocrinol 176: 575-582, 2017.

8. Papadakis GZ, Millo C, Sadowski SM, Bagci U and Patronas NJ: Kidney tumor in a von Hippel-Lindau (VHL) patient with intensely increased activity on ${ }^{68} \mathrm{Ga}$-DOTA-TATE PET/CT. Clin Nucl Med 41: 970-971, 2016.

9. El-Maouche D, Sadowski SM, Papadakis GZ, Guthrie L, Cottle-Delisle C, Merkel R, Millo C, Chen CC, Kebebew E and Collins MT: ${ }^{68} \mathrm{Ga}$-DOTATATE for tumor localization in tumor-induced osteomalacia. J Clin Endocrinol Metab 101: 3575-3581, 2016.

10. Papadakis GZ, Millo C, Sadowski SM, Bagci U and Patronas NJ: Epididymal cystadenomas in von Hippel-Lindau disease showing increased activity on ${ }^{68} \mathrm{Ga}$-DOTATATE PET/CT. Clin Nucl Med 41: 781-782, 2016.

11. Papadakis GZ, Millo C, Sadowski SM, Bagci U and Patronas NJ: Endolymphatic sac tumor showing increased activity on ${ }^{68}$ Ga-DOTATATE PET/CT. Clin Nucl Med 41: 783-784, 2016.

12. Papadakis GZ, Bagci U, Sadowski SM, Patronas NJ and Stratakis CA: Ectopic ACTH and CRH co-secreting tumor localized by ${ }^{68} \mathrm{Ga}$-DOTA-TATE PET/CT. Clin Nucl Med 40: 576-578, 2015

13. Papadakis GZ, Millo C, Karantanas AH, Bagci U and Patronas NJ: Avascular necrosis of the hips with increased activity on ${ }^{68} \mathrm{Ga}$-DOTATATE PET/CT. Clin Nucl Med 42: 214-215, 2017.

14. Papadakis GZ, Millo C, Bagci U, Sadowski SM and Stratakis CA: Schmorl nodes can cause increased ${ }^{68} \mathrm{Ga}$-DOTATATE activity on PET/CT, mimicking metastasis in patients with neuroendocrine malignancy. Clin Nucl Med 41: 249-250, 2016.

15. Li X, Samnick S, Lapa C, Israel I, Buck AK, Kreissl MC and Bauer W: ${ }^{68} \mathrm{Ga}$-DOTATATE PET/CT for the detection of inflammation of large arteries: Correlation with ${ }^{18} \mathrm{~F}-\mathrm{FDG}$, calcium burden and risk factors. EJNMMI Res 2: 52, 2012

16. Pedersen SF, Sandholt BV, Keller SH, Hansen AE, Clemmensen AE, Sillesen H, Højgaard L, Ripa RS and Kjær A: ${ }^{64} \mathrm{Cu}$-DOTATATE PET/MRI for detection of activated macrophages in carotid atherosclerotic plaques: Studies in patients undergoing endarterectomy. Arterioscler Thromb Vasc Biol 35: 1696-1703, 2015.

17. Tarkin JM, Joshi FR, Evans NR, Chowdhury MM, Figg NL, Shah AV, Starks LT, Martin-Garrido A, Manavaki R, Yu E, et al: Detection of atherosclerotic inflammation by ${ }^{68} \mathrm{Ga}$-DOTATATE PET compared to 18F-FDG PET imaging. J Am Coll Cardiol 69: 1774-1791, 2017.

This work is licensed under a Creative Commons Attribution-NonCommercial-NoDerivatives 4.0 International (CC BY-NC-ND 4.0) License. 